\title{
Grounding Orthodoxy and the Layered Conception*
}

\author{
Gabriel Oak Rabin
}

April 2016

\begin{abstract}
Ground offers the hope of vindicating and illuminating an classic philosophical idea: the layered conception, according to which reality is structured by relations of dependence, with physical phenomena on the bottom, upon which chemistry, then biology, and psychology reside. However, ground can only make good on this promise if it is appropriately formally behaved. The paradigm of good formal behavior can be found in the currently dominant grounding orthodoxy, which holds that ground is transitive, antisymmetric, irreflexive, and foundational. However, heretics have recently challenged the orthodoxy. In this paper, I examine ground's ability to vindicate the layered conception upon various relaxations of the orthodox assumptions. I argue that highly unorthodox views of ground can still vindicate the layered conception and that, in some ways, the heretical views enable ground to better serve as a guide to reality's layering than do orthodox views of ground.
\end{abstract}

${ }^{*}$ Thanks are due to two anonymous referees for extremely helpful comments and suggestions, and to Ricki Bliss and Graham Priest for inviting me to think about these topics and contribute to this volume. 


\section{Introduction}

Our world contains a shocking variety of stuff, from the very large (planets, quasars, galaxies) to the very small (quarks, leptons, bosons), with lots in between (koalas, canyons, coins). Here's a common thought: All this stuff can be organized into a hierarchy of levels. The galaxies and quasars are "on top", the canyons and koalas lie in the middle, below them come molecular compounds, and at the very bottom are the tiny particles and other phenomena (nuclear forces, electromagnetism) discussed in fundamental physics. The idea of "levels" in the special sciences reflects this hierarchical conception of the world. In the layering of special sciences, physics occupies the bottom, with chemistry, then biology, then psychology, then economics, lying on top.

What makes one phenomenon "higher" than another? One answer is that a relation of dependence creates the hierarchical structure. Psychology depends on biology, which depends on chemistry, which depends on physics. Of course, it's not the sciences themselves that depend on each other (psychology predates chemistry), but rather the phenomena the sciences study. Which psychological states I have depends on which biological states I have, but not vice versa. Which biological states I have depends on which chemical states I have, but not vice versa. Et cetera. Let's use the phrase 'the layered conception of reality' ('the layered conception' for short) as a label for the general idea that reality is layered in a hierarchy structured by relations of dependence. We can add a claim about fundamentality to the layered conception: the lower tiers of the layering are more fundamental than the higher tiers. I will make this further assumption in what follows.

Much philosophical ink has been recently spilled inquiring into the nature 
of ground. Ground is alleged to be a/the relation of metaphysical dependence, explanation, and/or priority. It is that relation the physicalist alleges to hold between the mental and the physical, that the utilitarian claims holds between moral facts and the facts about pleasure and pain, and that many claim to hold between the fact that $\mathrm{P}$ and the fact that $\mathrm{P}$ or $\mathrm{Q}$. In each case, the ground makes the grounded obtain. The grounded metaphysically depends on, is metaphysically explained by, and/or is ontologically posterior to, the ground. Ground should be distinguished from from causal dependence. Ground often (and perhaps always) holds synchronically, between two relata at the same time. For example, the physicalist claims that my current pain is grounded in my current brain state. In contrast, causal dependence relates items across time. The dualist can admit that my past brain state caused my current pain, while denying that pain is grounded in the brain. ${ }^{1}$

Once we have a notion of ground on board, it seems natural to slot that notion into the layered conception. After all, relations of dependence generate the layering, and ground is metaphysical dependence. Voila! Let's plug in everything we've learned in all the literature on ground to generate the layered picture of the world. Theorists of ground have had exactly this idea (deRosset [2013]). In fact, much of the appeal of the notion of ground, and its recent rise to prominency in metaphysics, comes from the intuitive appeal of the layered conception. Using ground to generate a hierarchy of dependence, and thereby vindicate the layered conception, is a nice thought, but it faces serious obstacles.

\footnotetext{
${ }^{1}$ I leave open the possibility that causation might, in the end, turn out to be a form of ground. Or vice versa. But prima facie, they look different, despite sharing some similarities.
} 
Only a relation with certain formal features is capable of delivering the layered conception of the world. For example, a layered hierarchy generated by a relation that loops will contain $\mathrm{X}$ above $\mathrm{Y}$, above $\mathrm{Z}$, but $\mathrm{X}$ will appear again down below Z! Loops aren't amenable to creating the type of structure characteristic of the layered conception. Thankfully, the orthodox views on ground hold that ground has several features that ensure that ground will be able to provide the structure characteristic of the layered conception. Let's label the conjunction of the following four theses 'the orthodoxy'. (All of these claims should be interpreted as preceded by universal quantifiers $\forall \mathrm{X}$, $\forall \mathrm{Y}, \forall \mathrm{Z}$. ):

(TS) Transitivity: If $\mathrm{X}$ grounds $\mathrm{Y}$ and $\mathrm{Y}$ grounds $\mathrm{Z}$, then $\mathrm{X}$ grounds $\mathrm{Z}$.

(AS) Antisymmetry: If $\mathrm{X}$ grounds $\mathrm{Y}$, then $\mathrm{Y}$ does not ground $\mathrm{X}$.

(IR) Irreflexivity: $\mathrm{X}$ does not ground $\mathrm{X}$.

(FD) Foundationalism: Everything is ultimately grounded in a bottom layer with no further ground. ${ }^{2}$

A relation that is transitive and antisymmetric cannot contain loops. This takes care of the worry that ground might generate loops, and thereby be unable to vindicate the layered conception. Or does it? The problem here is that every component of the orthodoxy has been challenged. Schaffer [2012] denies transitivity. Barnes [MS] denies antisymmetry. Jenkins [2011]

\footnotetext{
${ }^{2}$ This constraint sometimes goes under the banner that ground must be "well-founded" (Schaffer [2010]: 37). This is an unfortunate choice of terminology: a relation of ground that is not well-founded in the set-theoretic sense can still have a foundation. For clarification of these issues and of what "well-founded" amounts to when it comes to ground, cf. Rabin \& Rabern [2015].
} 
questions irreflexivity. Bliss [2014] even argues that ground might generate loops!

For the most part, theorists have either ignored the alleged counterexamples and continued to insist on the orthodoxy, or fought against the counterexamples outright (e.g. Litland [2013]). A major reason for maintaining the orthodoxy in the face of alleged counterexamples is the worry that without the formal features the orthodoxy provides, ground will provide unable to vindicate the layered conception. In the rest of this paper, my goal will be to alleviate this worry. I will argue that, even without any of the formal features listed above - transitivity, asymmetry, irreflexivity, or foundationalism - ground can still provide the dependence structure the layered conception requires. In fact, I will argue that relaxing the assumptions in the orthodoxy actually makes ground better able to generate the structure characteristic of the layered conception.

Here's a roadmap for the remainder of the paper. In the next section (2: "Ground as the Generator as Layers"), we put some flesh on the bones of the idea of the layered conception and how ground interacts with it. Each of sections 3-6 explores how ground fares in its ability to vindicate the layered conception under the relaxation of some element of the orthodoxy. We consider abandoning well-foundedness, anti-symmetry, irreflexivity, and transitivity (in that order). The conclusory Section 7 steps back to consider the resulting overall picture. 


\section{Ground as the Generator of Layers}

The layered conception is admittedly vague. In this section, we examine ways to put flesh on the bones of the bare idea and how we might utilize ground to elucidate the structure the layered conception mandates.

The layered conception, at first pass, looks something like this:

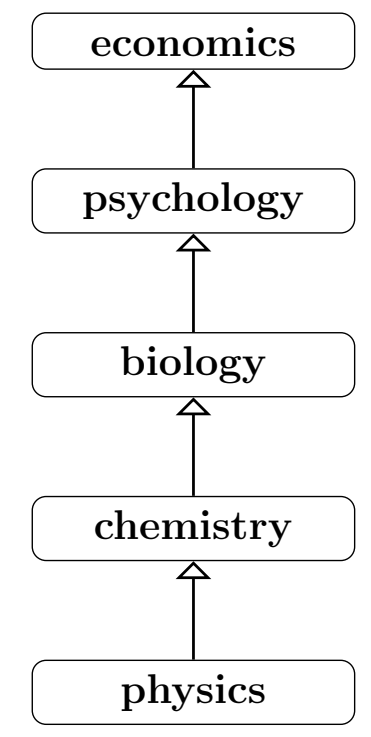

As I mentioned before, the claim is not that the sciences themselves, considered as fields of inquiry, depend on each other. Economists can and should go about their business without asking chemists for instructions. Instead, the phenomena studied by one field of inquiry depend on, and are determined by, phenomena studied by another field of inquiry. But that is not quite right. Biology depends on chemistry, but the camouflage in Arabian cuttlefish (a biological process) has absolutely nothing to do with the oxidization of steel beams (a chemical process) in a shipyard in New Orleans. Most particular concrete biological happenings have nothing to do with, and certainly don't 
depend on, most particular concrete chemical happenings. The same is true at the level of types. It's likely that the biological phenomenon of cuttlefish camouflage has nothing to with the chemical process of oxidization. (The marine biologists could prove me wrong here, but I feel like I'm on safe ground.)

However, the camouflage patterns of a particular cuttlefish do depend on some chemical facts about that particular cuttlefish. And the camouflage of a different cuttlefish depends on chemical facts about that cuttlefish. Furthermore, the two instances of cuttlefish camouflage might depend on the very same type (not token) of chemical property - call it ' $\mathrm{C}$ '. If the pattern is widespread, then we might claim a dependence of cuttlefish camouflage on chemical property C. This yields a lesson. We infer dependencies between types of properties from patterns in dependencies of particular tokens of those properties.

We now come to ground. Ground is typically understood as a dependence relation between particular facts, states of affairs, particulars, or properties. The mass of this table is grounded in the mass of these four legs and this table-top. Ground gives us the particular instances of dependency. From these particular token-dependencies we can infer the type-dependencies characteristic of the layered conception.

Sometimes, the type-dependencies are specific, such as when the firing of neurons is grounded in an electrical imbalance between positively charged potassium ions and negatively charged sodium. But these cases are rare. More often, the dependency is not specific, and a higher-level type, such as cuttlefish camouflage, does not depend on only one lower-level type, such 
as potassium/sodium interaction. In each particular case of cuttlefish camouflage, there is some chemical processes underlying it. But it needn't be the same type of chemical process in each case. These points are familiar from research on multiple realizability. Most phenomena are realizable, or groundable, in a wide variety of underlying lower-level phenomena. The various lower-level phenomena that all give rise to a single type of higher-level phenomenon might have little in common, other than the fact that they ground, or give rise to, the same type of higher-level happenings. Of course, these lower-level happenings, despite their dissimilarities, remain, e.g., chemical. So at the very least, we can say that cuttlefish camouflage, even if it does not depend on any particular chemical type, depends on "chemistry", or "the chemical level".

Call a complete story of the world's grounding relations between particular facts a grounding graph (so-called because it can be represented by a graph in the mathematical sense: a set of nodes with directed relations between them). The grounding graph gives us both more and less than we want from the layered conception. It gives us more because it gives us thousands of cuttlefish camouflage dependences - one for each cuttlefish. That's more than we need. But the grounding graph also gives us less. The layered conception says that biology is above chemistry. This entails that cuttlefish x's biological camouflage is above cuttlefish y's chemical properties. But grounding relations don't deliver this verdict. There are no grounding relations between the two. In mathematical terms, the layering conception seems to demand a total order, in which every pair of items is related by either the "higher than", "lower than", or "at the same level as" relation. In contrast, ground 
is a (very) partial order. A randomly chosen pair of items is unlikely to be related by ground at all. There's no easy recipe for generating a total order from a partial order.

However, there are reasons to be optimistic that the ordering characteristic of the layered conception can be gleaned from the grounding graph. First, as discussed above, we can look for patterns in the particular grounding claims. There are many such patterns. Sometimes the patterns are specific (neural firing depends on potassium-sodium ion imbalance). Other times they are not (each instance of cuttlefish camouflage depends on some chemical property). But the patterns are there. If they weren't, the layered conception wouldnt be so appealing in the first place. Second, we may not want the layered conception to deliver a total ordering. Both geology and psychology are above chemistry. Neither lies above the other. Two options remain: (i) they are at the same level or (ii) they are incommensurable.

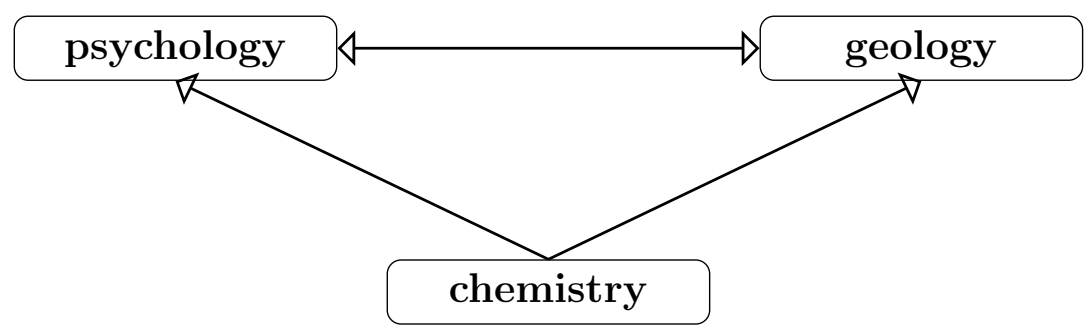

Figure 1: Option (i): geology and psychology on the same level, equally fundamental. 


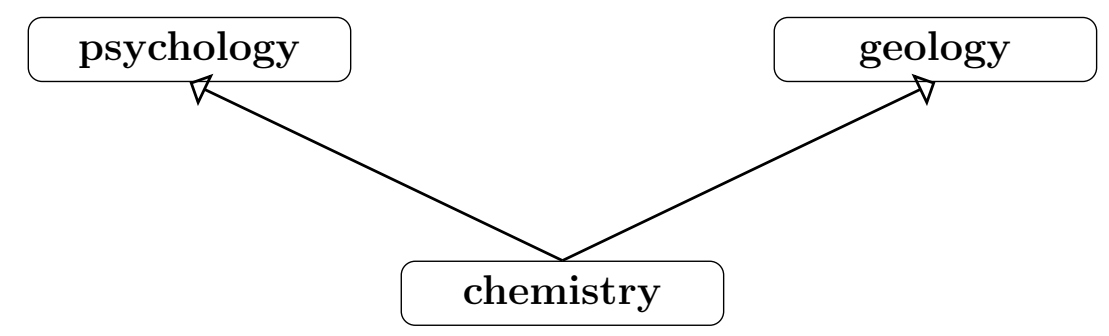

Figure 2: Option (ii): geology and psychology incommensurable, neither more, nor less, nor equally fundamental.

If the layered conception demands a total ordering, then (i) is the only option. A total ordering does not permit cases in which two items are incommensurable. However, I think that option (ii) is preferable, and that we should give up the idea that the layered conception requires a total ordering. Here's why. It remains open to discover some other range of phenomena, below psychology, but which contains no grounding relations to geology. Computation provides a potential example. If all psychological phenomena are ultimately grounded in computational phenomena (a not implausible hypothesis), then psychology will lie above computation. Suppose we choose option (i), placing geology on the same level with psychology. Ground tells us to place computation below psychology, which we've placed on the same level as geology. We're now forced to put computation below geology. This seems wrong. The relation between computational phenomena and geological phenomena is exactly the same as the relation between psychological phenomena and geological phenomena: nil. Whatever the reasons in favor of placing psychology and geology on the same level were, exactly the same reasons apply to placing computation and geology on the same level. It would be arbitrary to place geology and psychology on the same level with 
computation below, rather than, say, geology and computation on the same level, with psychology above.

The desire to place neither geology nor psychology above the other can be satisfied without placing them at the same level in the layered conception. Instead, we should give up the idea that the layered conception mandates a total ordering. Once we do so, ground, with its very partial order, looks better as a guide to reality's layers (as conceived by the layered conception). Admittedly, the layered conception demands an ordering that is closer to total than the ordering provided by ground. But patterns among ground's partial ordering can bridge the gap between grounds very partial order and the layered conception's less partial order.

\section{Foundationalism and the Layered Conception}

Foundationalism is the easiest bit of the orthodoxy for the fan of the layered conception to reject. Simply put, the layered conception does not require a foundation. The Greek philosopher Xenophanes was an early proponent of the layered conception (Patzia [n.d.], Lesher [1992]). Arguably, he also believed foundationalism to be false, and that the world consisted of alternating layers of earth and water. ${ }^{3}$

Of course, one could build foundationalism into the layered conception, forming the-layered-conception-with-a-bottom. In so doing, one would make the layered conception developed via ground incompatible with rejection of foundationalism about ground. But one certainly need not insist on a bottom

\footnotetext{
${ }^{3}$ Xenophanes believed in an infinite temporal descent of watery and earthy stages (of Rome [2015] attributes this view to Xenophanes in his Refutation of All Heresies: 1.14). Whether this entails anti-foundationalism of ground, will turn on whether temporal, or causal, dependence can be parlayed into metaphysical dependence.
} 
layer. The basic idea of a reality structured by relations of dependence does not require a foundation.

\section{Anti-symmetry and the Layered Conception}

The basic idea of using ground to generate the layered conception comes from the following principle:

(The Simple Principle) If $\mathrm{x}$ grounds $\mathrm{y}$, then $\mathrm{x}$ is at a lower level / more fundamental than $\mathrm{y}$.

The simple principles gets us from claims about grounding relations between particulars (facts, objects, or properties) to claims about where those particulars fit into reality's layers. To generate the full layered conception, we still need to discern patterns concerning where certain types of things occur in reality's layers. But, via the simple principle, ground gives us a good start.

The simple principle does not work so well, however, if ground fails to be antisymmetric. According to the simple principle, if $\mathrm{x}$ grounds $\mathrm{y}$, then $\mathrm{x}$ is lower than $\mathrm{y}$. If $\mathrm{y}$ grounds $\mathrm{x}$ (violating anti-symmetry), then $\mathrm{y}$ is lower than $\mathrm{x}$. And that doesn't make sense, at least in so far as I understand the layered conception. Biology can’t be both above and below chemistry.

There are decent prima facie considerations in favor of rejecting the antisymmetry of ground. Barnes [MS] argues that we should accept symmetric dependence in a wide variety of cases, from immanent universals to states of affairs to mathematical ontology. In one example, she argues that it is essential to the evacuation at Dunkirk that it is part of World War II. And it is essential to World War II that it contain the evacuation at Dunkirk. If 
this is correct, it is plausible to maintain that each of World War II and the evacuation at Dunkirk depend on the other. Voila: symmetric ground!

This is neither the time nor the place to have the fight over whether ground is or is not antisymmetric. Barnes presents some plausible cases. At the least, proponents of the theory-combinations Barnes discusses might want to take advantage of a non antisymmetric (i.e. sometimes symmetric) notion of ground. For their sake, it's worth exploring how rejecting the orthodoxy regarding the anti-symmetry of ground interacts with the layered conception.

I believe that, ultimately, rejection of anti-symmetry for ground does not impugn ground's ability to vindicate the layered conception. In fact, cases of symmetric ground might help us better understand how reality is layered. I argue for these claims in the remainder of this section.

The simple principle, above, is one way to infer layering from relations of ground. But once we recognize the possibility of symmetric ground, we can opt for the following slightly less simple principle.

(The Slightly Less Simple Principle) If $\mathrm{x}$ grounds $\mathrm{y}$, and y does not ground $\mathrm{x}$, then $\mathrm{x}$ is more fundamental / at a lower level than $\mathrm{y}$.

The Slightly Less Simple Principle is a clear improvement over the Simple Principle. If ground is antisymmetric, then the ' $y$ does not ground $x$ ' clause in the Less Simple Principle is vacuous, and the Less Simple Principle reduces to the Simple Principle. But if symmetric ground does occur, the Slightly Less Simple Principle avoids the problematic result above, where $\mathrm{x}$ is both above and below y in reality's layering.

In cases of symmetric ground, what should we say about the layering relations of the items that ground each other? We should not place either 
above the other. This leaves two options, which we've already seen: (i) they are at the same level or (ii) they are incommensurable. I believe that (i) is the better option here. $\mathrm{x}$ and $\mathrm{y}$ are related by ground. It seems quite odd to say that they bear no relation to each other in reality's layering. The layering is still a layering based on dependence. And $\mathrm{x}$ and $\mathrm{y}$ depend on each other. I propose we place $\mathrm{x}$ and $\mathrm{y}$ on the same level.

Considerations involving the transitivity of ground further support placing $\mathrm{x}$ and $\mathrm{y}$ on the same level. The transitivity of ground will guarantee that, in cases of symmetric ground, the symmetric grounders will be at the pseudo-same level. For any $\mathrm{x}$ and $\mathrm{y}, \mathrm{x}$ and $\mathrm{y}$ are at the pseudo-same level in reality's layering if and only if for any $\mathrm{z}$, if $\mathrm{z}$ is above $\mathrm{x}$, then $\mathrm{z}$ is above $\mathrm{y}$, and if $\mathrm{z}$ is below $\mathrm{x}$, then $\mathrm{z}$ is below $\mathrm{y}$. In simple terms, two items at the pseudosame level are both above, and below, all the same stuff. This does not quite guarantee sameness of level. $\mathrm{x}$ and $\mathrm{y}$ might still be incommensurable.

It is worth noting that this case is slightly different than geology-biology case discussed earlier, in which I argued for incommensurability of level. In that case, computation lied below biology, but remained incommensurable with geology. This would not be possible if geology and biology were incommensurable but at the pseudo-same level. Their pseudo-sameness would guarantee that if biology were higher than computation, geology would be too.

I admit that my arguments leave some space for claiming that symmetric grounders are incommensurable in level. But given that (a) they are related by dependence and (b) they are at the pseudo-same level, I believe we should say that they lie at the same level in reality's layering. 


\section{$5 \quad$ Irreflexivity and the Layered Conception}

Grounding orthodoxy holds that ground is irreflexive: nothing grounds itself. Jenkins [2011] has challenged the orthodoxy, claiming that it's better to leave open the possibility that something could ground itself. For example, an identity theorist in philosophy of mind might simultaneously claim that (a) consciousness is identical to electrical flow in the brain's dorsal stream and (b) mental phenomena, including consciousness, are grounded in brain phenomena, such as electrical flow in the dorsal stream. If the orthodoxy is correct, this position is incoherent: ground is irreflexive. Consciousness can't be grounded in electrical flow in the dorsal stream, to which it is identical.

Understandably, Jenkins argues that our conception of ground should not rule out by fiat the combination of metaphysical views espoused by the envisioned identity theorist. We want ground to provide a useful philosophical tool for conceptualizing various debates in metaphysics. In so far as an irreflexive conception of ground makes unintelligible a plausible and popular view in the philosophy mind, it fails to accomplish this goal. The best solution, argues Jenkins, is to give up the irreflexivity of ground. The result is not that ground is reflexive (i.e. everything grounds itself), but that sometimes, things do ground themselves.

There are ways to resist this line of thought. But acceptance, in certain cases, of reflexive ground, seems desirable. Particularly so in light of particular philosophical views, like the identity theory in philosophy of mind. How much does giving up the irreflexivity of ground affect ground's ability to vindicate the layered conception? The answer is, "Not much."

In combination with the simple principle, cases of irreflexive ground cause 
problems. Continuing with the identity claim as our example, the two will entail that conscious experience is above itself (and below itself) in the layered conception. That's weird.

Like with symmetric ground, a shift from the simple principle to the slightly less simple principle saves the day. The slightly less simple principle avoids the result that conscious experience is above (and below) itself in reality's layering.

The choice between an irreflexive conception of ground and a reflexive conception might be partly terminological. In the semantics of Fine [2012], weak ground, in which everything grounds itself, is taken as the primitive notion. Fine does this partly for reasons of simplicity. But we might think that formal simplicity provides some reason for taking the reflexive conception of ground to be more fundamental, even if talk of an entity's grounding itself rubs against thought of ground as a form of metaphysical explanation and/or determination.

One important difference between giving up irreflexive ground and giving up antisymmetric ground is worth noting. If ground is reflexive, i.e. if everything grounds itself, there is no serious challenge to the layered conception. We need simply shift from the unreflective simple principle to the slight less simple principle. Such a move will avoid the unsavory implications of cases of reflexive ground (e.g. that conscious experience is both above and below itself), but still allow ground to play its intended role in generating the remainder of reality's layering. On the other hand, if ground is symmetric, i.e. if every time $\mathrm{x}$ grounds $\mathrm{y}, \mathrm{y}$ grounds $\mathrm{x}$, the goal of using ground to generate reality's layering falls into serious jeopardy. There's no simple fix for 
symmetric ground. (Thankfully, to my knowledge no one has suggested that ground is symmetric).

The basic thought behind using ground to generate the layered conception is that if $\mathrm{x}$ grounds $\mathrm{y}, \mathrm{x}$ is lower than $\mathrm{y}$ in the layered conception. In the first instance, ground relates tokens, or particular facts. The layered conception relates types (as well as tokens of those types). Some theorizing is required to get from the tokens to the types. Adoption of a reflexive conception of ground, in which everything grounds itself, requires only minimal modification of the basic idea. A shift from the basic idea, expressed in the simple principle, to a more nuanced version of the same idea via the slightly less simple principle, does the trick and rescues a reflexive conception of ground's ability to generate reality's layering.

In contrast, symmetric ground, in which every time $\mathrm{x}$ grounds $\mathrm{y}$, $\mathrm{y}$ also grounds $\mathrm{x}$, completely voids the basic idea. Ground will never give us the result that $\mathrm{x}$ is above (or below) $\mathrm{y}$ in reality's layering. In the previous section, I argued that in cases of symmetric ground we should maintain that the symmetric groundees should be placed at the same level in reality's layering. If this is correct, then ground will provide some, but not much, guide to reality's layers. Ground will be sufficient for sameness of level. But some other relation will be required to do the heavy lifting in the generation of reality's vertical hierarchy.

In sum, I claim that neither acceptance of particular cases of reflexive ground nor acceptance of a fully reflexive conception of ground seriously challenges the ability of ground to vindicate the layered conception. Particular individual cases of symmetric ground can be easily handled. But a full-blown 
symmetric conception of ground will void ground's ability to provide reality's layering.

\section{Intransitivity and the Layered Conception}

Lastly, we come to the transitivity of ground, which says that if $\mathrm{x}$ grounds y, and y grounds z, then x grounds z. Schaffer [2012] has challenged this principle. One of his arguments revolves around a dented sphere. Schaffer claims that while it's plausible that (a) the fact that the dented sphere has a dent grounds that fact that it has determinate shape $S$ and (b) the fact that the dented sphere has determinate shape $\mathrm{S}$ grounds the fact that it is more-or-less spherical, it is implausible that (c) the fact that the dented sphere has a dent grounds the fact that it is more-or-less spherical. After all, writes Schaffer, "the thing is more-or-less spherical despite the minor dent, not because of it." (127).

There are ways to resist the argument, but I do not wish to weigh in on the issue here. Schaffer's example is prima facie plausible, and he provides other alleged counterexamples to transitivity. At the least, some will want to deny the transitivity of ground. For their sake, it's worth exploring how such a denial will affect ground's interaction with the layered conception.

The layered conception's hierarchical structure is transitive. If biological phenomena lie above chemical phenomena, and psychological phenomena lie above the biological, then psychological phenomena lie above chemical phenomena. We need some transitivity in the layered conception.

Consider a graphical representation of the world's grounding relations, in which nodes represent the relata of grounding relations and arrows between 
nodes represent relations of ground. (Arrows point from the ground to the grounded).

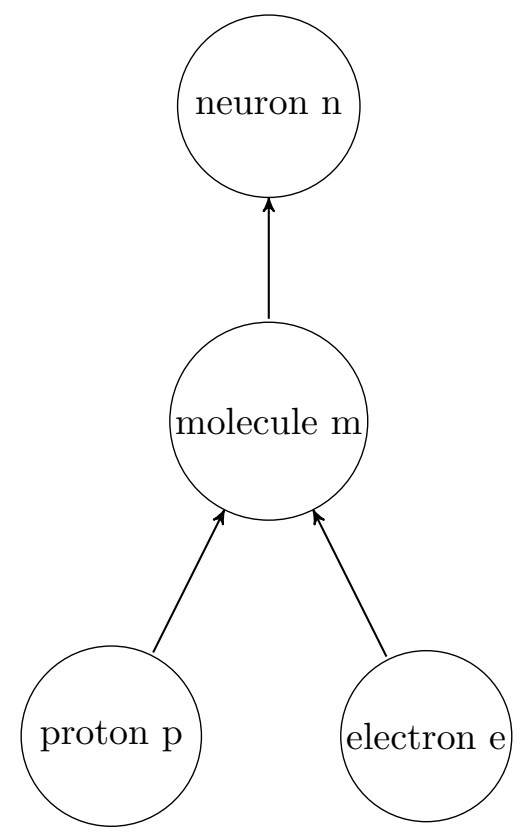

From the graph, we can observe the beginnings of reality's layering. The fact that my brain contains proton $\mathrm{p}$ lies below the fact that my brain contains potassium molecule $\mathrm{m}$, which lies below the fact that my brain contains neuron $\mathrm{n}$. This layering of particular facts proceeds from the physical to the chemical to the biological. The generation of this layering does not require an arrow, or a relation of ground, between proton $\mathrm{p}$ and neuron $\mathrm{n}$ or their associated facts. A failure of transitivity, say, between the proton and the neuron, will not interfere with the generation of this layering.

From a formal standpoint, this should be no surprise. For any nontransitive relation $\mathrm{R}$ one can always take the transitive closure of $\mathrm{R}$ to generate a transitive relation $R^{*}$ that will contain $R$ as a subset, in the sense that 
if Rab, then $\mathrm{R}^{*} \mathrm{ab}$. Even if ground is not transitive, we can take ground's transitive closure to generate ground*. But we need not resort to such formal tricks.

The layered conception involves a layering of fundamentality. The chemical is more fundamental than the biological. "More fundamental than" is transitive, as are "higher than" and "lower than" in reality's layering. Ground and fundamentality are linked by the simple and/or slightly less principle we've discussed. Grounding relations have implications for relations of relative fundamentality and for reality's layering. But ground can fail to be transitive, and even be anti-transitive, yet still have these implications for the transitive relations for "more/less fundamental than" and "lower/higher in reality's layering than". Assuming this transitivity, a double application of the simple (and/or slightly less simple) principle yields the result that if $\mathrm{x}$ grounds $\mathrm{y}$, and $\mathrm{y}$ grounds $\mathrm{z}$, then $\mathrm{x}$ is more fundamental than $\mathrm{y}$, and $\mathrm{x}$ is lower than $\mathrm{z}$ in reality's layering. This is so even if $\mathrm{x}$ does not ground $\mathrm{z}$.

One final worry goes as follows. If ground is not transitive, but the hierarchical structure of the layered conception is, what is the layered conception a hierarchy of? The preceding discussion should alleviate the worry. The layered conception's hierarchical structure captures relations of relative fundamentality, which have an intimate relation to ground, despite the fact that they remain transitive even when ground is not.

We can get the transitive structure constitutive of the layered conception even if ground fails to be transitive. The transitivity can come in later, with the relations ("more/less fundamental than", "lower/higher than") that properly constitute reality's layering, and to which ground is a guide. 


\section{Conclusion}

The key to making unorthodox views about the formal properties of ground compatible with the layered conception is to recognize that there is a gap between what grounds what and the layered conception. One can't just "read off" reality's layering from the facts about ground. The move from what grounds what to reality's layering is substantive. I believe we should be optimistic about gleaning from the facts about ground a useful and informative structure that roughly matches our pre-theoretic conception of how the features of reality are layered.

First, principles linking ground and layering, or fundamentality, such as the simple and/or slightly less simple principle, give us a healthy start in generating a layering from ground. But the task of evaluating the patterns in the grounding relations between particulars, and gleaning from those patterns a layering of the various properties, and types of properties (geological, biological), remains. Second, we may have to abandon some of our pre-theoretic ideas about reality's layering. I argued that we should abandon the claim that reality's layering generates a total order. Geology and biology are incommensurable; neither lies above or below the other. The layering's order is closer to total than ground's order. But both are partial.

The gap between ground and layering both helps and harms. It harms because it makes the task of discerning reality's layering more difficult. Even after we possess a complete story of what grounds what, we must still do philosophical work to determine what is more fundamental than what. It helps because it permits the layering to be well-behaved even when ground is

not. For example, symmetric cases of ground don't force us to claim that the 
symmetric groundees each lie above (or below) the other in reality's layering.

The grounding orthodoxy ensures that ground behaves nicely. It will be a good little transitive, antisymmetric, irreflexive, well-founded relation. This obedient behavior ensures the absence of problematic grounding structures, such as loops, that create problems when we move from ground to reality's layering. But the heretics are out there. Not all theorists of ground believe in the orthodoxy. I've covered a variety of reasons to doubt various parts of that orthodoxy. These theorists will probably be willing to give up some nice behavior in order to have a theoretical tool that can do the metaphysical work they want done. For this reason alone, it's worth exploring how reality's layering might go if we accept an unorthodox view about ground and want to maintain an intimate link between ground the layered conception.

There are good reasons for the orthodoxy. The principles seem prima facie correct. It's convenient to have a formally well-behaved relation. But there are good reasons to doubt the orthodoxy. Cases like Jenkins' reflexive dependence of pains on brains, or Barnes' symmetric dependence of World War II and the evacuation at Dunkirk, should force us to seriously reconsider. There is something to the idea of mutual dependence in those cases. This dependence should at least be taken into consideration when we move to generate reality's layering. A non-orthodox conception of ground will better reflect whatever it was about dependence that Barnes and Jenkins latched on to, and which we want reflected in the reality's layering. Even staunchly orthodox views, when they move from the grounding graph to reality's layering, might decide to reflect that symmetric relation in reality's layering, even if they do not choose to call it 'ground'. In this way a non-orthodox 
conception of ground better reflects reality's relations of dependence, and enables the generation of a more, rather than less, accurate, picture of reality's layering.

In the end, we might reject the arguments of Barnes, Bliss, Jenkins, and Schaffer, and maintain that the orthodoxy about ground is correct. But knowing that the layered conception is perfectly compatible with the heretical views that challenge the orthodoxy should grease the wheels for rejecting that orthodoxy (a move with which I have considerable sympathy). A nonorthodox view of ground can not only have a nice layering of reality, but the non-orthodox view is, in various ways, better suited to that layering. The grounding heretics can have their (layered) cake and eat it too.

\section{References}

Barnes, Elizabeth. MS. Symmetric Dependence.

Bliss, Ricki. 2014. Viciousnesss And Circles Of Ground. Metaphilosophy, 45, $245-56$.

deRosset, Louis. 2013. Grounding Explanations. Philosophers' Imprint.

Fine, Kit. 2012. A Guide to Ground. In: Correia, Fabrice, \& Schneider, Benjamin (eds), Metaphysical Grounding: Understanding the Structure of Reality. Cambridge: Cambridge University Press.

Jenkins, Carrie. 2011. Is Metaphysical Dependence Irreflexive? The Monist, 94, 267-76.

Lesher, J. H. 1992. Xenophanes of Colophon: Fragments: A Text and Translation with Commentary. University of Toronto Press.

Litland, Jon. 2013. On Some Counterexamples To The Transitivity of Grounding. Philosophical Essays, 14, 19-32. 
of Rome, Hippolytus. 2015. Refutation of All Heresies. CreateSpace Independent Publishing Platform.

Patzia, Michael. Xenophanes. The Internet Encyclopedia of Philosophy.

Rabin, Gabriel, \& Rabern, Brian. 2015. Well Founding Grounding Grounding. Journal of Philosophical Logic, 1-25.

Schaffer, Jonathan. 2010. Monism: The priority of the whole. The Philosophical Review, 119(1), 31.

Schaffer, Jonathan. 2012. Grounding, Transitivity, and Contrastivity. Pages 122-138 of: Correia, Fabrice, \& Schneider, Benjamin (eds), Metaphysical Grounding: Understanding the Structure of Reality. 